\title{
Vaccines as Tools to Reduce ARI Burden: Merits and Some Inherent Verticality in the Debate
}

\author{
SANJAY Chaturvedi \\ Professor of Community Medicine, University College of Medical Sciences and GTB Hospital, Delhi, India. \\ Correspondence to: Dr Sanjay Chaturvedi, E-15, GTB Hospital Campus, Dilshad Garden, Delhi 110 095, India. \\ cvsanjay@hotmail.com
}

$\mathrm{A}$

cute respiratory infections (ARI) continue to stay among the main causes of child mortality. Even in the general

population, they are the leading cause of acute morbidities globally. Developing countries are the worst sufferers where they top the list of causes of disability adjusted life years (DALYs) lost. Estimates suggest that the incidence density of ARI in underfive children among developing and industrialized world is 0.29 and 0.05 episodes per child-year, accounting for 151 million and 5 million new episodes each year, respectively. India (43 million), China (21 million), Pakistan (10 million), and Bangladesh (5.6 million) bear the maximal brunt(1). Pneumonia, influenza, tuberculosis, and bronchiolitis are the chief contributors to ARI related mortality for it is the lower respiratory infection (LRI) that leads to severe forms of clinical presentations. Most vulnerable to develop a fatal ARI are very young, elderly and the immunocompromized and in this context, a child with suboptimal immunity for any reason is doubly at risk. While early detection and treatment with antimicrobials significantly reduces mortality, the advantage is blunted by lack of access to care, treatment delays and treatment failures because of rising resistance to commonly used antimicrobials. Preventive modalities like immunization, improved nutrition and reduced indoor air pollution offer a proactive and cost-effective line of intervention(2). Tuberculosis, for its inherent chronicity and BCG vaccine, for a plethora of rather noisy evidence, deserve a dedicated space. Here, we restrict ourselves to certain innovations in the existing immunization program as well as some newer vaccines to be used for a sustained reduction in the incidence of ARI.

The chief pathogens responsible for ARI in children are Streptococcus pneumoniae, Haemophilus influenzae type $b$ (Hib), Staphylococcus aureus, respiratory syncytial virus (RSV), measles virus, human influenza virus $\mathrm{A} / \mathrm{H} 1 \mathrm{~N} 1$, and parainfluenza viruses (PIV-1, PIV-2 and PIV-3). Influenza virus has an added threat of novel strains causing pandemic waves - presently novel A/H1N1 with a potential threat of A/H5N1. Of these, licensed vaccines recommended by both the global as well as national expert bodies are available against measles, Hib, Streptococcus pneumoniae, and seasonal human influenza $\mathrm{A} / \mathrm{H} 1 \mathrm{~N} 1$. One opportunity for the measles containing vaccine (MCV-1) is a part of national immunization schedule since mid eighties, and soon the Hib vaccine may join the universal immunization programme (UIP) as an ingredient of multi-dose liquid pentavalent vaccine (DPT-HepBHib)(3). Availability of acellular pertussis (aP) has added to UIP options. Among the non-UIP group, pneumococcal vaccines are making a very strong case for inclusion and influenza vaccines are seeking immediate attention.

\section{UIP VACCINES}

The fact file of measles in India raises several 
concerns. Our surveillance is largely outbreakcentric and the mortality associated with measles gets underrepresented for even in the health system data and verbal autopsies, several cases are recorded as pneumonia deaths. With the rejuvenated efforts to eliminate measles, India is the only country in the region not offering the second opportunity for measles containing vaccine (MCV-2). Now, the National Technical Advisory Group on Immunization (NTAGI) has recommended its delivery through supplementary immunisation activities (SIA) for children aged 9 months to 10 years in the states with $\geq 80 \% \mathrm{MCV}-1$ coverage; and through measles-rubella (MR) or measles-mumps-rubella (MMR) vaccine during second year of life in the states with sustained $\geq 80 \% \mathrm{MCV}-1$ coverage( 4 ). The strategies are technically correct but in view of the fatigue and social resistance seen with polio eradication initiative, any campaign mode SIA may place the measles elimination program in a social and political hotspot. The phenomenology illustrated by qualitative evidence from western Uttar Pradesh may have a bearing on the measles-centric verticality as well(5). Ordinary people have something very meaningful to share with us. Some of them are shouting from the rooftop but we are reluctant to listen. If we do, we would realize that populationbased disease control activities are much more than a technical mission. It is being argued that the phenomenal social mobilization and energized health machinery of pulse polio should be exploited for measles agenda as well. Under the pressure of international advocacy and the cultural verticality of national experts, we may continue to ignore the voices of most peripheral health workers and people.

The scheduling of MCV-1 at 9 months has an ethical issue also that we have been ignoring for a decade. A small proportion of measles infection is borne by children between 6 to 9 months. These young children suffer with the disease before reaching the eligibility for immunization. In developing countries, maternal antibody levels against measles may be low and severe outbreaks of measles can occur in younger infants. The standard Edmonston-Zagreb vaccine that we have today has shown $94 \%$ protective efficacy even among infants aged 4.5 months(6). This age group may look small in size but ethically, we are denying vaccination to some children at a higher risk. Epidemiologically too, this constituency will become significant as we approach elimination. Immediate goal of high coverage should not be delinked from the need for timely immunization. There is a case for advancing the age of first opportunity for measles vaccination, not as substitute but as supplement to the vaccine given at 9-15 months(7).

Acellular pertussis (aP) vaccines containing purified antigens are better tolerated than whole-cell vaccines (wP), and are now used in all regions of the world. Both the types have comparable efficacy and it would be prudent to gradually replace $\mathrm{wP}$ with aP. The higher cost of aP may be the only impeding factor(8). Since the immunity after early childhood immunization with DTwP/DTaP wanes over a decade and evidence from industrialized countries has shown a rising secular trend of pertussis in adolescents and adults, a single dose of Tdap (standard quantity tetanus toxoid, reduced quantity diphtheria toxoid and three acellular pertussis components in reduced quantities viz. pertussis toxoid, filamentous hemagglutinin and pertactin) is being recommended at 10-12 years instead of $\mathrm{Td}$ alone(9).

\section{NON-UIP V ACCINES}

The conjugate Hib vaccines have demonstrated safety and efficacy world over, including India, and there is a global consensus that lack of local surveillance data should not delay the introduction of these vaccines in all infant immunization schedules(10). They have also demonstrated a substantive herd effect. NTAGI was concerned about reports of adverse events following immunization (AEFI) from Sri Lanka but the investigations conducted by WHO did not reveal any causal association between the events and the Hib containing vaccine(4). Hib vaccines are available as monovalent, tetravalent (DTwP-Hib) and pentavalent (DTwP-HepB-Hib) formulations, and in similar combinations with triple vaccine containing acellular pertussis (DTaP). They fit into the 6-10-14 week primary vaccination schedule followed by a booster at 15-18 months with triple vaccine and oral polio. Several Indian manufacturers are producing 
Hib vaccines and with sufficient lead time, would be able to meet the domestic demand by scaling up the production(11). The planned nationwide roll out of pentavalent vaccine by 2012 is supported by sound arguments and evidence(3). However, it may be logistically challenging to launch it across the country in a single phase. States with high under-5 mortality should be given priority, especially the districts with low routine immunization (RI) coverage. This will help the most vulnerable children both by the direct and herd effect even if there is no drastic improvement in the RI. A close monitoring of roll out projects and adverse events following immunisation (AEFI) would ensure the managerial and technical correctness of the initiative but carefully planned strategies for communication and partnership with people and civil society, especially involving non-utilizer clients, are going to be critical.

Pneumococcal vaccines are knocking at the door for quite sometime. Both the unconjugated polysaccharide vaccine (PPV) as well as conjugate pneumococcal vaccines (PCV) are available. PPV, a 23 valent vaccine, has low efficacy and does not induce herd immunity. Poor immunogenicity of this vaccine among the under- 2 children, who are at higher risk for pneumococcal disease, was the primary reason why conjugate PCVs were developed(12). Nevertheless, PPV 23 has retained its relevance for its broad serotype coverage and is recommended to be administered with PCV in high risk children. The 7 valent pneumococcal conjugate vaccine (PCV 7) containing polysaccharide antigen of serotypes 4, 6B, 9V, 14, 18C, 19F and 23 linked to a protein carrier has shown remarkable safety and efficacy. The vaccine has also demonstrated some herd effect resulting from reduced nasopharyngeal carriage(9). Evidence of protection from other pneumococcal illnesses like meningitis and otitis media is not robust as yet, and on the safety front there have been some concerns about the possible rise in reactive airway disease. Based on experience from over 25 developed countries and risk-benefit ratio, WHO has recommended its inclusion in the national immunization programs on a priority basis, especially in countries with above 50 under-5 mortality rate(13). PCV 9, PCV 10 and PCV 13 are also in the advanced stages of trials. In the case of PCV too, the 6-10-14 week routine immunization and a booster at 15-18 months fits well with the UIP schedule. Since the maximal force of infection and virulence of pneumococcal disease is borne by underprivileged and high risk children, the PCV should be offered to them first. Cost should not be used as a deterrent - and it is not that prohibitive either. GAVI has offered to supply PCV at a cost of Rs. 15/dose, till 2015(9).

The major concern is the coverage of circulating serotypes in India. In the evolving scenario of serotype composition of PCVs, NTAGI has rightly applied a cautious approach. The group has recommended that the vaccine to be introduced in the national program should cover more than $70 \%$ of the circulating serotypes in India. PCV7 offers only $55 \%$ coverage in this regard. It also needs to be explored whether this can be achieved with minimal number of serotypes included in the vaccine. That would be the appropriate product to be piloted. NTAGI has also recommended that the indigenous manufacturers should be encouraged to develop such a vaccine(4). This will reduce the cost and make the program self reliant in post 2015 scenario. An assessment of joint introduction of Hib vaccines with newer 10 and 13 valent PCVs for mortality impact would answer several questions, as both are effective against pneumonia and meningitis $(4,11)$. Advocacy to establish Hib and pneumococcal disease surveillance system for baseline measurement of disease and impact monitoring has its technical merit but when seen with the similar demands for a verticality in the surveillance of other important diseases like measles, the proposal does not look feasible. Although many feel that the present format of Integrated Disease Surveillance Project (IDSP) does not suitably inform immunization program, the general consensus favors the integrated approach to surveillance. Newer information needs of UIP will have to be woven around an augmented IDSP.

Seasonal influenza is neither a serious child health problem in India nor there are clearly defined influenza seasons across the country - inspite of winter peaks in the north(14). What brings urgency to this agenda is the ongoing pandemic caused by 
novel A/H1N1. Vaccination against seasonal human influenza is a relatively complex process. The subjects are to be immunized every year with a newer vaccine. This process of constant change is informed by a global surveillance of viral drift. Both killed and live attenuated vaccines are available but only the killed vaccine is licensed in India. Both have good safety records but their efficacy in India would only be based on an informed guess. Current evidence does not suggest that seasonal influenza vaccination either decreases or increases the risk for acquiring pandemic H1N1 illness(15). Pandemic vaccines from several manufacturers are now available within the developed world. Although all of them are based on the initial isolate A/California/ 7/2009(H1N1), they are likely to be effective since no significant genetic or antigenic drift has occurred till now(16). With present constraints and availability, the pandemic vaccine can only be recommended to groups at very high risk. In-country production of pandemic vaccine, considered by NTAGI, may not be a pragmatic response to emerging situation. Haridwar Maha-Kumbh 2010, the biggest human congregation on earth, coinciding with the dreaded second wave in the northern hemisphere, is already on and by the time we have an indigenous vaccine -6 months after having new isolate, it may be too little, too late. Nonpharmaceutical interventions would be the only preventive modality available in large parts of India(17). We can only hope that the second wave is as muted as its predecessor. Many argue that the global antigenic surveillance machinery is tuned to inform vaccine needs for North America and Europe, and even if some reference laboratories in the developing world detect a local strain, it will have a low probability of inclusion in the newer vaccine. These concerns get neutralized by the communicability of influenza virus added with the level of civil aviation that we have today. Seasonal, pre-pandemic or pandemic, the idea of having a region specific vaccine would be self defeating. The challenges posed by any local drift would be instantly made global by today's civil aviation. The developed countries know it fully well.

All this debate and advocacy on inclusion of newer vaccines to child immunization schedule is to be seen in the context of weak infrastructure of primary care delivery and poor RI coverage. Several undocumented reports of non-inclusion of sizeable areas in the RI microplanning also need to be factored here. With only $43.5 \%$ fully immunized children - and serious rural-urban, inter-state and inter-district divide in this 'documented' universe and percentages $(18,19)$ - widening the spectrum of diseases to be covered by available UIP and non-UIP vaccines would not only result into suboptimal impact but may also end up highlighting the health inequities across class, strata and regions. Indiscriminate advertisements of several vaccines by the manufacturers, often promoted by reach and credibility of child health clinics, also needs to be regulated. Although cutting edge technology and equity in health care are not mutually exclusive, one may be forced to ask - will this public health grammar always favor the class that has power to access and purchase? An argument that sounds specious from this side of the table may prove quite robust from the other side. Even a vaccine made universally available may continue to deny several children's right to get immunized. Access remains a result of privileges.

Funding: None.

Competing interests: None stated.

\section{REFERENCES}

1. World Health Organization. Acute Respiratory Infections (Update September 2009). http:// www.who.int/vaccine_research/diseases/ari/en/ print.html. Accessed 4 November, 2009.

2. Niessen L, Hove AT, Hilderink H, Weber M, Mulhollande K, Ezzati M. Comparative impact assessment of child pneumonia interventions. Bull World Health Organ 2009; 87: 472-480.

3. WHO India. Pneumonia a major killer in children; prevention possible with safe \& effective vaccines. http://www.whoindia.org/EN/Section6/ Section453.htm. Accessed 4 November, 2009.

4. Ministry of Health and Family Welfare, Govt. of India. Minutes of the meeting of the National Technical Advisory Group on Immunization (NTAGI), New Delhi: 3 Aug 2009.

5. Chaturvedi S, Dasgupta R, Adhish V, Ganguly KK, Rai S, Sushant L, et al. Deconstructing social 
resistance to pulse polio campaign in two North Indian Districts. Indian Pediatr 2009; 46: 963-974.

6. Martins CL, Garly ML, Balé C, Rodrigues A, Ravn $\mathrm{H}$, Whittle HC, et al. Protective efficacy of standard Edmonston-Zagreb measles vaccination in infants aged 4.5 months: interim analysis of a randomised clinical trial. BMJ 2008; 337: a661.

7. Broutin H, Miller MA. Early vaccination against measles in developing countries. BMJ 2008; 337: a406.

8. World Health Organization. WHO position on pertussis vaccines. Wkly Epidemiol Rec 2005; 80: 37-39.

9. Indian Academy of Pediatrics Committee on Immunization (IAPCOI). Consensus Recommendations on Immunization, 2008. Indian Pediatr 2008; 45: 635-648.

10. World Health Organization. WHO position paper on Haemophilus influenzae type b conjugate vaccines. Wkly Epidemiol Rec 2006; 81: 445-452.

11. Subcommittee on Introduction of Hib Vaccine in Universal Immunization Program, National Technical Advisory Group on Immunization, India. NTAGI subcommittee recommendations on Haemophilus influenzae Type $\mathrm{b}$ (Hib) vaccine introduction in India. Indian Pediatr 2009; 46: 945-954.

12. Advisory Committee on Immunization Practices. Preventing pneumococcal disease among infants and young children. Recommendations of the
Advisory Committee on Immunization Practices (ACIP). MMWR Recomm Rep 2000; 49 (RR-9): 1-35.

13. World Health Organization. Pneumococcal conjugate vaccine for childhood immunization WHO position paper. Wkly Epidemiol Rec 2007; 82: 93-104.

14. Mathew JL. Influenza vaccination for children in India. Indian Pediatr 2009; 46: 304-307.

15. Centers for Disease Control and Prevention (CDC). Effectiveness of 2008-09 trivalent influenza vaccine against 2009 pandemic influenza A (H1N1) - United States, May-June 2009. MMWR Morb Mortal Wkly Rep 2009; 58:1241-1245.

16. Johansen K, Nicoll A, Ciancio BC, Kramarz P. Pandemic influenza A(H1N1) 2009 vaccines in the European Union. Euro Surveill 2009; 14: 19361.

17. Chaturvedi S. Pandemic influenza: imminent threat, preparedness and the divided globe. Indian Pediatr 2009; 46: 115-121.

18. Ministry of Health and Family Welfare, Govt. of India. National family health survey, 2005-2006 (NFHS-3): National fact sheet. http:// www.nfhsindia.org/pdf/IN.pdf. Accessed 4 November, 2009.

19. Ministry of Health and Family Welfare, Govt. of India. District level household and facility survey, 2007-2008 (DLHS-3). http://www.rchiips.org/ state-fact-sheet-rch3.html. Accessed 4 November, 2009. 